\title{
Necessary Conditions for Optimization in Multiparameter Discrete Systems ${ }^{1}$
}

\author{
D. R. HEGG ${ }^{2}$ AND N. H. MCClAmROCH \\ Communicated by W. F. Powers \\ Dedicated to L. Cesari
}

\begin{abstract}
A general first-order dynamic representation for discrete systems with several independent variables is proposed, based on the Dieudonné-Rashevsky form for partial differential equations. This representation does not restrict consideration to causal systems. A minimum principle for such systems is proved, thus extending results known for discrete-time systems to the case of several independent variables. The proof requires only the classical implicit function theorem.
\end{abstract}

Key Words. Discrete models, partial difference equations, Dieudonné-Rashevsky form, noncausal systems, necessary conditions, derived cones, separation of convex sets, implicit function theorem.

\section{Introduction}

The widespread availability of high-speed digital computers has sparked a renewal of interest in the use of difference equations for modeling dynamic systems. At the same time, researchers in diverse scientific disciplines are realizing that, in many cases, difference equations give a much more accurate representation of system dynamics than differential equations. Numerous authoritative treatises defending the use of discrete mathematical models have appeared (Refs. 1-3), as have sophisticated

\footnotetext{
${ }^{1}$ This research was performed at the University of Michigan and was supported in part by Grants Nos. NGR-23-005-017 and NGR-23-005-131 from the National Aeronautics and Space Administration.

${ }^{2}$ Member, Technical Staff, Charles Stark Draper Laboratory, Cambridge, Massachusetts.

${ }^{3}$ Professor, Department of Aerospace Engineering and Program in Computer, Information, and Control Engineering, University of Michigan, Ann Arbor, Michigan.
} 
analyses of the dynamics of discrete models (Refs. 4-6). Moreover, advances in electronics are creating new dynamic systems for which a continuum mathematical model is decidedly inappropriate (Ref. 7). Until very recently, research in discrete system theory focused on systems with only one independent variable. However, the capability for high-speed digital signal processing (in particular, of two-dimensional images) has generated much interest in the development both of discrete models in several (in particular, two) independent variables (Refs. 8-10) and of associated discrete multiparameter system theory (Ref. 11).

In this paper, a general model for discrete multiparameter systems consisting of first-order nonlinear partial difference equations is proposed. This model is a discrete form of the Dieudonné-Rashevsky (DR) equations used by Cesari (Ref. 12) and Suryanarayana (Ref. 13) in optimization studies with partial differential equations. The model has descriptor form (see Ref. 14), making explicit a property used by Cesari and Suryanarayana without comment. Most systems of partial difference equations encountered in applications (including noncausal systems) can be placed in this discrete DR form (Ref. 15). In particular, most state-space system models recently developed for linear two-parameter discrete (2-D) systems (Refs. 16-18) can be placed in this form.

The main contribution of this paper is the statement and proof of necessary conditions for optimal control of multiparameter systems in the discrete DR form. The approach to the proof is motivated by the work of Baum and Cesari (Ref. 19) in simplifying the proof of the Pontryagin maximum principle. A notable feature is that only the classical implicit function theorem (rather than a fixed-point theorem) is required. The theory of optimization for discrete-time systems is mature (Refs. 20-25). Optimization with multiparameter systems involving discrete variables has proceeded in several directions with respect to the type of models used: discretization in time only (e.g., Ref. 26); discretization in space variables only (e.g., Ref. 27); and discretization in all variables (Refs. 28-31). Results in the present paper extend to fully discrete multiparameter systems the sharpest general minimum principle known for discrete-time systems (Ref. 25).

\section{Optimization Problem}

Problem Definition. The optimization problem is in Mayer form with partial difference equations and static constraints defined over a finite rectangular array of multiindices. Given positive integers $m$ and $k_{\alpha}, \alpha=$ $1, \ldots, m$, denote by $G$ the Cartesian product (array) $\prod_{\alpha=1}^{m}\left\{0, \ldots, k_{\alpha}\right\}$, and 
by $k$ the corner point $\left(k_{1}, \ldots, k_{m}\right)$. For each index $\alpha$, denote by $1^{\alpha}$ the unit displacement $(0, \ldots, 0,1,0, \ldots, 0)$ in the $\alpha$ th variable, and by $G_{k}(\alpha)$ the subset

$$
\left\{i \equiv\left(i_{1}, \ldots, i_{m}\right) \in G: i_{\alpha} \neq k_{\alpha}\right\} .
$$

Assume that the following are specified: additional positive integers $s_{\alpha}$, $\alpha=1, \ldots, m$, and $n$; a nonempty set $U_{0}$; a family of subsets $U(i), i \in G \sim k$, of $U_{0}$ (the symbol $\sim$ denotes set-theoretic difference); a family of subsets $X(i), i \in G$, of Euclidean space $E^{n}$; a family of real matrices $S^{\alpha}(i): s_{\alpha} \times n$, $i \in G_{k}(\alpha), \alpha=1, \ldots, m$; a family of functions $f^{\alpha}(i, \cdot, \cdot): E^{n} \times U_{0} \rightarrow E^{s_{\alpha}}$, $i \in G_{k}(\alpha), \alpha=1, \ldots, m$; and a function $f_{0}: E^{n} \rightarrow E^{1}$. A pair $(x, u)$, with $x: G \rightarrow E^{n}$ and $u: G \sim k \rightarrow U_{0}$ is called admissible if it satisfies:

$$
\begin{array}{cl}
S^{\alpha}(i) x\left(i+1^{\alpha}\right)=f^{\alpha}(i, x(i), u(i)), & i \in G_{k}(\alpha), \alpha=1, \ldots, m, \\
u(i) \in U(i), & i \in G \sim k, \\
x(i) \in X(i), \quad & i \in G .
\end{array}
$$

The optimization problem is to minimize the functional

$$
(x, u) \mapsto I(x, u) \triangleq f_{0}(x(k))
$$

over the class of admissible pairs. A minimizing pair is called optimal.

Discrete System Model. Equations (1) constitute the discrete Dieudonné-Rashevsky form for multiparameter partial difference equations. Its relationship with proposed multiparameter state-space models for linear systems is briefly indicated for the 2-D case. Kung et al. (Ref. 32) have shown that the Roesser model (Ref. 17) is the most general of the models proposed in Refs. 16-18 (see Ref. 33 for an alternative viewpoint). Consider the $2-\mathrm{D}$ Roesser model with horizontal and vertical local states $x_{1}: n_{1} \times 1$ and $x_{2}: n_{2} \times 1$, respectively (using the notation of the preceding paragraph),

$$
\begin{array}{ll}
x_{1}(i+(1,0))=A_{1} x_{1}(i)+B_{1} x_{2}(i)+C_{1} u(i), & i \equiv\left(i_{1}, i_{2}\right) \in G_{k}(1), \\
x_{2}(i+(0,1))=A_{2} x_{1}(i)+B_{2} x_{2}(i)+C_{2} u(i), & i \in G_{k}(2) .
\end{array}
$$

See Ref. 17 for the distinction between local and global states in 2-D systems. These equations are easily expressed in the form (1) for the vector

$$
x \triangleq\left(x_{1}, x_{2}\right)^{T}
$$

by using

$$
S^{1} \triangleq\left[\begin{array}{cc}
I_{n_{1}} & 0 \\
0 & 0
\end{array}\right] \text { and } S^{2} \triangleq\left[\begin{array}{cc}
0 & 0 \\
0 & I_{n_{2}}
\end{array}\right]
$$


Here, $I_{\rho}$ denotes the identity matrix, and the superscript $T$ denotes matrix transpose. Recently, Chan (Ref. 34) has introduced a new multiparameter state-space model in which more general parameters replace the unit shifts $1^{\alpha}$. This model can be translated into a form similar to Eqs. (1) if Chan's generalized shifts are used in (1) instead of the shifts $1^{\alpha}$. More importantly, it should be observed that the proposed form (1) is not restricted to use with causal systems as are all of the local state-space models. Some of the fundamental difficulties associated with the development of multiparameter discrete linear system theory are outlined in Ref. 35. However, a discussion of these matters, as they relate to the linear form of Eqs. (1), is beyond the scope of the present paper.

\section{Necessary Conditions}

Preliminaries. Several definitions facilitate the statement of results. With each point

$$
i \equiv\left(i_{1}, \ldots, i_{m}\right)
$$

of the array $G$ are associated the index sets

$$
\Gamma^{j}(i) \triangleq\left\{\alpha \in\{1, \ldots, m\}: i_{\alpha} \neq j_{\alpha}\right\}, \quad j=0, k ;
$$

and with each index $\alpha$ in $\{1, \ldots, m\}$ is associated the subset

$$
G_{0}(\alpha) \triangleq\left\{i \equiv\left(i_{1}, \ldots, i_{m}\right) \in G: i_{\alpha} \neq 0\right\} .
$$

The boundary of $G$ is the set

$$
B \triangleq\left\{i \equiv\left(i_{1}, \ldots, i_{m}\right) \in G: \exists \alpha \in\{1, \ldots, m\} \text { with } i_{\alpha} \in\left\{0, k_{\alpha}\right\}\right\} .
$$

A Hamiltonian function, defined for $i$ in $G \sim k, x$ in $E^{n}, u$ in $U(i)$, $p^{\alpha}$ in $E^{s_{\alpha}}, \alpha=1, \ldots, m$, is given by

$$
H\left(i, x, u, p^{1}, \ldots, p^{m}\right) \triangleq \sum_{\alpha \in \Gamma^{k}(i)} p^{\alpha} \cdot f^{\alpha}(i, x, u) .
$$

Here, the symbol $\cdot$ denotes scalar product.

Given a nonempty set $A$ in $E^{n}$ and a point $x$ of $A$, a closed convex cone $C$ is said to be a derived cone for $A$ at $x$ if, for each finite subset $h_{\rho}$, $\rho=1, \ldots, r$, of $C$, there exists a continuously differentiable map $\sigma:[0,1]^{r} \rightarrow E^{n}$ such that:

(i) $\sigma(t) \in A$, for each $t \equiv\left(t_{1}, \ldots, t_{r}\right)$ in $[0,1]^{r}$;

(ii) $\sigma(0)=x$;

(iii) $\left(\partial \sigma / \partial t_{\rho}\right)(0)=h_{\rho}, \rho=1, \ldots, r$.

The polar of a closed convex cone in $E^{n}$ is denoted by the superscript $^{\circ}$. 
Theorem 3.1. Minimum Principle. Assume the following: $X(k)$;

(H1) $f_{0}$ is continuously differentiable on (i.e., in a neighborhood of)

(H2) for each triple $\alpha$ in $\{1, \ldots, m\}, i$ in $G_{k}(\alpha), u$ in $U(i)$, the map $f^{\alpha}(i, \cdot, u)$ is continuously differentiable on $X(i)$;

(H3) for each pair $i$ in $G \sim k, x$ in $X(i)$, the set $f(i, x, U(i))$, where $f$ denotes $\left(f^{\alpha}\right)_{\alpha \in \Gamma^{k}(i)}$, is convex.

Let $\left(x^{*}, u^{*}\right)$ be an optimal pair; and, for each $i$ in $G$, let $C(i)$ be a derived cone for $X(i)$ at $x^{*}(i)$.

Then, there exist $p_{0} \geq 0$ and vectors $p^{\alpha}: G_{k}(\alpha) \rightarrow E^{s_{\alpha}}, \alpha=1, \ldots, m$, satisfying:

(a) $\left(p_{0}, p^{1}, \ldots, p^{m}\right) \neq 0$

(b) $\sum_{\alpha=1}^{m} S^{\alpha^{T}}\left(i-1^{\alpha}\right) p^{\alpha}\left(i-1^{\alpha}\right)$

$-(\partial H / \partial x)^{T}\left(i, x^{*}(i), u^{*}(i), p^{1}(i), \ldots, p^{m}(i)\right) \in[C(i)]^{\circ}, i \in G \sim B ;$

(c) $H\left(i, x^{*}(i), u^{*}(i), p^{1}(i), \ldots, p^{m}(i)\right)$

$=\min _{u \in U(i)}\left\{H\left(i, x^{*}(i), u, p^{1}(i), \ldots, p^{m}(i)\right)\right\}, i \in G \sim k ;$

(d) $\sum_{\alpha \in \Gamma^{\mathrm{b}}(i)} S^{\alpha^{T}}\left(i-1^{\alpha}\right) p^{\alpha}\left(i-1^{\alpha}\right)$

$-(\partial H / \partial x)^{T}\left(i, x^{*}(i), u^{*}(i), p^{1}(i), \ldots, p^{m}(i)\right) \in[C(i)]^{\circ}, i \in B \sim k ;$

$\sum_{\alpha=1}^{m} S^{\alpha^{T}}\left(k-1^{\alpha}\right) p^{\alpha}\left(k-1^{\alpha}\right)-p_{0}\left(\partial f_{0} / \partial x\right)^{T}\left(x^{*}(k)\right) \in[C(k)]^{\circ}$.

The sum over the empty set that occurs in (d) is assigned the value zero.

\section{Proof of Necessary Conditions} where

Motivation. Note that Eqs. (1) are equivalent to $\gamma$ scalar equations,

$$
\gamma \stackrel{\Delta}{=} \sum_{\alpha=1}^{m} s_{\alpha} \operatorname{card} G_{k}(\alpha)
$$

The notation $\left(z_{i \alpha}\right)_{i \alpha}$ is used to denote vectors $\left(z_{i \alpha}\right)_{i \in G_{k}(\alpha)}, \alpha=1, \ldots, m$, in $E^{\gamma}$. Consider the set $\mathscr{P}$ of elements $\left(z_{0},\left(z_{i \alpha}\right)_{i \alpha}\right)$ in the augmented space 
$E^{\gamma+1}$ defined by the conditions

$$
\begin{gathered}
z_{0}=f_{0}(x(k))-f_{0}\left(x^{*}(k)\right), \\
z_{i \alpha}=f^{\alpha}(i, x(i), u(i))-S^{\alpha}(i) x\left(i+1^{\alpha}\right), \quad i \in G_{k}(\alpha), \alpha=1, \ldots, m, \\
u(i) \in U(i), \quad i \in G \sim k, \\
x(i) \in X(i), \quad i \in G,
\end{gathered}
$$

where $\left(x^{*}, u^{*}\right)$ represents an admissible pair, and also the set

$$
\mathscr{Q} \triangleq\left\{(-\eta, 0, \ldots, 0) \in E^{\gamma+1}: \eta>0\right\} .
$$

Disjointness of $\mathscr{P}$ and $\mathscr{Q}$ characterizes optimality of a pair $\left(x^{*}, u^{*}\right)$. Proof of the necessary conditions follows from establishing the weaker condition that $\mathscr{Q}$ is algebraically separated from a suitable convex approximation to $\mathscr{P}$.

Proof. For each triple $\alpha$ in $\{1, \ldots, m\}, i$ in $G_{k}(\alpha), u$ in $U(i)$, denote

$$
\begin{aligned}
A^{\alpha}(i) & \triangleq\left(\partial f^{\alpha} / \partial x\right)\left(i, x^{*}(i), u^{*}(i)\right), \\
F^{\alpha}(i, u) & \triangleq f^{\alpha}\left(i, x^{*}(i), u\right)-f^{\alpha}\left(i, x^{*}(i), u^{*}(i)\right),
\end{aligned}
$$

and also denote

$$
a_{0} \triangleq\left(\partial f_{0} / \partial x\right)^{T}\left(x^{*}(k)\right)
$$

Denote by $\mathscr{R}$ the set of points $\left(z_{0},\left(z_{i \alpha}\right)_{i \alpha}\right)$ in $E^{\gamma+1}$ defined by the conditions

$$
\begin{aligned}
z_{0}=a_{0} \cdot h(k), & \\
z_{i \alpha}=A^{\alpha}(i) h(i)+F^{\alpha}(i, u(i))-S^{\alpha}(i) h\left(i+1^{\alpha}\right), & i \in G_{k}(\alpha), \alpha=1, \ldots, m,
\end{aligned}
$$

$$
\begin{array}{ll}
u(i) \in U(i), & i \in G \sim k, \\
h(i) \in C(i), & i \in G .
\end{array}
$$

In view of the hypotheses, it follows immediately that $\mathscr{R}$ is convex and contains the origin. To show that $\mathscr{2}$ and $\mathscr{R}$ are separated, two cases are distinguished, depending on whether or not $\mathscr{R}$ has (topological) interior points. The interior of $\mathscr{R}$, denoted by $\operatorname{int}(\mathscr{R})$, is convex since $\mathscr{R}$ is convex.

Separation of $\mathscr{Q}$ and $\mathscr{R}$. If $\mathscr{R}$ has empty interior, then the linear manifold in $E^{\gamma+1}$ generated by $\mathscr{R}$ is a proper affine space that can be extended to a hyperplane, say $p \cdot z=\beta$, for some nonzero $p$ in $E^{\gamma+1}$; the first component, say $p_{0}$, of $p$ can be assumed nonnegative. Since $\mathscr{R}$ contains 
the origin and lies in the hyperplane, it follows that $\beta=0$. Then, for each $z \equiv(-\eta, 0, \ldots, 0)$ in 2 ,

$$
p \cdot z=-p_{0} \eta \leq 0,
$$

which establishes separation for this trivial case.

If $\mathscr{R}$ has nonempty interior, it can be shown that $\mathscr{Q}$ and the interior of $\mathscr{R}$ are disjoint. Verification of this assertion entails technical arguments peripheral to the main thrust of the proof, and is therefore postponed to later in this section. Assuming that this assertion is true, it follows from well-known separation results that $\mathscr{Q}$ and $\operatorname{int}(\mathscr{R})$ are separated by a hyperplane, say $p \cdot z=\beta(p \neq 0)$; in particular,

$$
\begin{array}{ll}
p \cdot z \geq \beta, & \text { for } z \text { in } \operatorname{int}(\mathscr{R}), \\
p \cdot z \leq \beta, & \text { for } z \text { in } \mathscr{Q} .
\end{array}
$$

But, since int $(\mathscr{R})$ is nonempty,

$$
\operatorname{cl}(\mathscr{R})=\operatorname{cl}(\operatorname{int}(\mathscr{R})),
$$

where $\operatorname{cl}(\cdot)$ denotes the closure (Ref. 36$)$. Hence, $\mathscr{R}$ itself lies in the closed half-space

$$
p \cdot z \geq \beta
$$

Denote by $p_{0}$ the first component of $p$, and let $\eta>0$ be arbitrary. Since the zero vector is in $\mathscr{R}$, and since $(-\eta, 0, \ldots, 0)$ is in $\mathscr{Q}$, the inequalities

$$
p_{0}(-\eta) \leq \beta \leq 0
$$

hold. Hence,

$$
p_{0} \geq 0 \text {. }
$$

Moreover, since $\eta$ is arbitrary, $\beta$ must be zero.

Deduction of the Necessary Conditions. The preceding subsection shows that $\mathscr{R}$ lies in the half-space

$$
p \cdot z \geq 0,
$$

defined by a nonzero vector $p \equiv\left(p_{0},\left(p^{\alpha}(i)\right)_{i \alpha}\right)$ satisfying

$$
p_{0} \geq 0 \text {. }
$$

This proves assertion (a).

Let $u: G \sim k \rightarrow U_{0}$ and $h: G \rightarrow E^{n}$ satisfy Eqs. (4) and (5), respectively, but be otherwise arbitrary. This defines a general point of $\mathscr{R}$ given by Eqs. 
(2) and (3). The separation inequality for such a point of $\mathscr{R}$ is the following:

$$
\begin{aligned}
p_{0} a_{0} & \cdot h(k)+\sum_{\alpha=1}^{m} \sum_{i \in G_{k}(\alpha)} A^{\alpha^{T}}(i) p^{\alpha}(i) \cdot h(i) \\
& +\sum_{\alpha=1}^{m} \sum_{i \in G_{k}(\alpha)} p^{\alpha}(i) \cdot F^{\alpha}(i, u(i)) \\
& -\sum_{\alpha=1}^{m} \sum_{i \in G_{k}(\alpha)} S^{\alpha^{T}}(i) p^{\alpha}(i) \cdot h\left(i+1^{\alpha}\right) \geq 0 .
\end{aligned}
$$

Following some algebra, this inequality may be rewritten in a form that isolates the free parameter vectors $u(\cdot)$ and $h(\cdot)$ :

$$
\begin{aligned}
\sum_{i \in \mathcal{G} \sim B}\{ & \left.\sum_{\alpha=1}^{m}\left[S^{\alpha}\left(i-1^{\alpha}\right) p^{\alpha}\left(i-1^{\alpha}\right)-A^{\alpha^{T}}(i) p^{\alpha}(i)\right]\right\} \cdot h(i) \\
& +\sum_{i \in B \sim\{0 . k\}}\left[\sum_{\alpha \in \Gamma^{(0)}(i)} S^{\alpha^{T}}\left(i-1^{\alpha}\right) p^{\alpha}\left(i-1^{\alpha}\right)\right. \\
& \left.-\sum_{\alpha \in \Gamma^{k}(i)} A^{\alpha^{T}}(i) p^{\alpha}(i)\right] \cdot h(i) \\
& -\sum_{\alpha \in \Gamma^{k}(0)}\left[A^{\alpha^{T}}(0) p^{\alpha}(0)\right] \cdot h(0) \\
& +\left[\sum_{\alpha=1}^{m} S^{\alpha^{T}}\left(k-1^{\alpha}\right) p^{\alpha}\left(k-1^{\alpha}\right)-p_{0} a_{0}\right] \cdot h(k) \\
\leq & \sum_{i \in \mathcal{G} \sim k} \sum_{\alpha \in \Gamma^{k}(i)} p^{\alpha}(i) \cdot F^{\alpha}(i, u(i)) .
\end{aligned}
$$

Assertions (b)-(d) are deduced by writing Ineq. (6) with several judicious choices of the free parameter vectors.

First, choose

$$
h(i) \triangleq 0, \quad i \in G,
$$

select arbitrary points $i$ in $G \sim k$ and $w$ in $U(i)$, and choose for $u$ the mapping

$$
u(\lambda) \triangleq \begin{cases}u^{*}(\lambda), & \lambda \in G \sim\{k, i\}, \\ w, & \lambda=i .\end{cases}
$$

With this choice of free parameter vectors, Ineq. (6) reduces to

$$
\sum_{\alpha \in \Gamma^{k}(i)} p^{\alpha}(i) \cdot F^{\alpha}(i, w) \geq 0,
$$

which is equivalent to assertion (c). 
Next, choose

$$
u(i) \triangleq u^{*}(i), \quad i \in G \sim k,
$$

select arbitrary points $i$ in $G$ and $\zeta$ in $C(i)$, and choose for $h$ the mapping

$$
h(\lambda) \triangleq \begin{cases}0, & \lambda \in G \sim i \\ \zeta, & \lambda=i .\end{cases}
$$

With this choice of free parameter vectors, Ineq. (6) reduces to either

$$
\begin{gathered}
\left\{\sum_{\alpha=1}^{m}\left[S^{\alpha^{T}}\left(i-1^{\alpha}\right) p^{\alpha}\left(i-1^{\alpha}\right)-A^{\alpha^{T}}(i) p^{\alpha}(i)\right]\right\} \cdot \zeta \leq 0, \\
{\left[\sum_{\alpha \in \Gamma^{\alpha}(i)} S^{\alpha^{T}}\left(i-1^{\alpha}\right) p^{\alpha}\left(i-1^{\alpha}\right)-\sum_{\alpha \in \Gamma^{k}(i)} A^{\alpha^{T}}(i) p^{\alpha}(i)\right] \cdot \zeta \leq 0,} \\
-\left[\sum_{\alpha \in \Gamma^{k}(0)} A^{\alpha^{T}}(0) p^{\alpha}(0)\right] \cdot \zeta \leq 0,
\end{gathered}
$$

or

$$
\left[\sum_{\alpha=1}^{m} S^{\alpha^{T}}\left(i-1^{\alpha}\right) p^{\alpha}\left(i-1^{\alpha}\right)-p_{0} a_{0}\right] \cdot \zeta \leq 0,
$$

according as the point $i$ selected lies in

(i) $G \sim B$,

(ii) $B \sim\{0, k\}$,

(iii) $\{0\}$,

(iv) $\{k\}$,

respectively. Since $\zeta$ is an arbitrary element of $C(i)$, Ineqs. (7)-(10) imply, respectively, that

$$
\begin{aligned}
& \sum_{\alpha=1}^{m}\left[S^{\alpha^{T}}\left(i-1^{\alpha}\right) p^{\alpha}\left(i-1^{\alpha}\right)-A^{\alpha^{T}}(i) p^{\alpha}(i)\right] \in[C(i)]^{\circ}, \\
& \sum_{\alpha \in \Gamma^{\alpha}(i)} S^{\alpha T}\left(i-1^{\alpha}\right) p^{\alpha}\left(i-1^{\alpha}\right)-\sum_{\alpha \in \Gamma^{k}(i)} A^{\alpha^{T}}(i) p^{\alpha}(i) \in[C(i)]^{0}, \\
- & \sum_{\alpha \in \Gamma^{k}(0)} A^{\alpha^{T}}(0) p^{\alpha}(0) \in[C(i)]^{\circ},
\end{aligned}
$$

or

$$
\sum_{\alpha=1}^{m} S^{\alpha^{T}}\left(i-1^{\alpha}\right) p^{\alpha}\left(i-1^{\alpha}\right)-p_{0} a_{0} \in[C(i)]^{0},
$$

which are equivalent, collectively, to assertions (b) and (d). The proof is complete, except for the verification that 2 and int $(\mathscr{R})$ are disjoint. 
Disjointness of $\mathscr{2}$ and int $(\mathscr{R})$. The proof is by contraposition. Suppose that

$$
z \equiv\left(z_{0},\left(z_{i \alpha}\right)_{i \alpha}\right) \in \mathscr{Q} \cap \operatorname{int}(\mathscr{R}) .
$$

It will be shown that this implies that $\left(x^{*}, u^{*}\right)$ cannot be optimal. On the one hand, since $z \in \mathscr{Q}$, it follows that

$$
\eta \stackrel{\Delta}{\underline{\Delta}}-z_{0}>0
$$

and

$$
z_{i \alpha}=0, \quad \text { for each } i \text { in } G_{k}(\alpha), \alpha=1, \ldots, m .
$$

On the other hand, since $z \in \operatorname{int}(\mathscr{R})$, there is an open set $N$ in $E^{\gamma+1}$ with $z \in N \subset \mathscr{R}$. Consider the vectors

$$
e^{\rho} \triangleq(0, \ldots, 0,1,0, \ldots, 0), \quad \rho=1, \ldots, \gamma,
$$

in $E^{\gamma+1}$, with the unit in the $(\rho+1)$ th coordinate for each, and the sum vector

$$
b \triangleq \sum_{\rho=1}^{\gamma} e^{\rho} .
$$

Then, there exists $\delta>0$ such that the simplex with vertices $z+\delta e^{\rho}, \rho=$ $1, \ldots, \gamma$, and $z-\delta b$ lies inside $N$, and hence in $\mathscr{R}$. Using Eqs. (2)-(5), it follows that:

(i) for each $\rho=1, \ldots, \gamma$, there exist $u^{\rho}: G \sim k \rightarrow U_{0}$ and $h^{\rho}: G \rightarrow E^{n}$ such that

$$
z_{0}=a_{0} \cdot h^{\rho}(k)
$$

$$
\begin{aligned}
\left(z_{i \alpha}\right)_{i \alpha}+(0, \ldots, 0, \delta, 0, \ldots, 0) & \\
= & \left(A^{\alpha}(i) h^{\rho}(i)+F^{\alpha}\left(i, u^{\rho}(i)\right)-S^{\alpha}(i) h^{\rho}\left(i+1^{\alpha}\right)\right)_{i \alpha,}, \\
u^{\rho}(i) & \in U(i), \quad i \in G \sim k, \\
h^{\rho}(i) & \in C(i), \quad i \in G,
\end{aligned}
$$

with each $\delta$ appearing, respectively, in the $\rho$ th coordinate;

(ii) there exist $u^{\gamma+1} ; G \sim k \rightarrow U_{0}$ and $h^{\gamma+1}: G \rightarrow E^{n}$ such that

$$
\begin{gathered}
z_{0}=a_{0} \cdot h^{\gamma+1}(k), \\
\left(z_{i \alpha}\right)_{i \alpha}+(-\delta, \ldots,-\delta)=\left(A^{\alpha}(i) h^{\gamma+1}(i)+F^{\alpha}\left(i, u^{\gamma+1}(i)\right)-S^{\alpha}(i) h^{\gamma+1}\left(i+1^{\alpha}\right)\right)_{i \alpha}, \\
u^{\gamma+1}(i) \in U(i), \quad i \in G \sim k, \\
h^{\gamma+1}(i) \in C(i), \quad i \in G .
\end{gathered}
$$


Comparison of the two expressions for the components of $z$ shows that

$$
\begin{gathered}
a_{0} \cdot h^{\rho}(k)=-\eta, \quad \rho=1, \ldots, \gamma+1, \\
\left(A^{\alpha}(i) h^{\rho}(i)+F^{\alpha}\left(i, u^{\rho}(i)\right)-S^{\alpha}(i) h^{\rho}\left(i+1^{\alpha}\right)\right)_{i \alpha} \\
=\left\{\begin{array}{cc}
(0, \ldots, 0, \delta, 0, \ldots, 0), & \rho=1, \ldots, \gamma, \\
(-\delta, \ldots,-\delta), & \rho=\gamma+1 .
\end{array}\right.
\end{gathered}
$$

Let $i \in \mathrm{G}$ be arbitrary. Since $h^{\rho}(i) \in C(i), \rho=1, \ldots, \gamma+1$, and since $C(i)$ is a derived cone for $X(i)$ at $x^{*}(i)$, there exists a continuously differentiable $C^{1}$-map $\sigma(i ; \cdot):[0,1]^{\gamma+1} \rightarrow E^{n}$ such that

$$
\begin{aligned}
& \sigma(i ; t) \in X(i), \quad \text { for each } t \equiv\left(t_{1}, \ldots, t_{\gamma+1}\right) \text { in }[0,1]^{\gamma+1}, \\
& \sigma(i ; 0)=x^{*}(i),
\end{aligned}
$$

and

$$
\left(\partial \sigma / \partial t_{\rho}\right)(i, 0)=h^{\rho}(i), \quad \rho=1, \ldots, \gamma+1 .
$$

The map $\sigma(i ; \cdot)$ can be extended to a $C^{1}$-map $\hat{\sigma}(i ; \cdot)$ on some neighborhood of $[0,1]^{\gamma+1}$. Let $D_{1}$ be a neighborhood of $[0,1]^{\gamma+1}$ in $E^{\gamma+1}$ on which each extension $\hat{\sigma}(i ; \cdot), i \in G$, is $C^{1}$. For each triple $t \equiv\left(t_{1}, \ldots, t_{\gamma+1}\right)$ in $D_{1}$, $\alpha$ in $\{1, \ldots, m\}, i$ in $G_{k}(\alpha)$, define $y^{\alpha}\left(i+1^{\alpha} ; t\right)$ as the sum

$$
\left[1-\sum_{\rho=1}^{\gamma+1} t_{\rho}\right] f^{\alpha}\left(i, \hat{\sigma}(i ; t), u^{*}(i)\right)+\sum_{\rho=1}^{\gamma+1} t_{\rho} f^{\alpha}\left(i, \hat{\sigma}(i ; t), u^{\rho}(i)\right) .
$$

For $t=0$, it follows from Eqs. (1) that

$$
y^{\alpha}\left(i+1^{\alpha} ; 0\right)=S^{\alpha}(i) x^{*}\left(i+1^{\alpha}\right), \quad i \in G_{k}(\alpha), \alpha=1, \ldots, m .
$$

For $t$ satisfying

$$
t_{\rho} \geq 0, \quad \rho=1, \ldots, \gamma+1, \quad \sum_{\rho=1}^{\gamma+1} t_{\rho} \leq 1,
$$

then

$$
\hat{\sigma}(i ; t)=\sigma(i ; t) \in X(i), \quad \text { for each } i \text { in } G .
$$

Hypothesis (H3) of Theorem 3.1 ensures that each of the sets $f(i, \sigma(i ; t)$, $U(i)), i \in G \sim k$, is convex. Hence, there exist maps $u(\cdot ; t): G \sim k \rightarrow U_{0}$, with $u(i ; t) \in U(i)$, for each $i$ in $G \sim k$, such that

$$
y^{\alpha}\left(i+1^{\alpha} ; t\right)=f^{\alpha}(i, \sigma(i: t), u(i ; t)), \quad i \in G_{k}(\alpha), \alpha=1, \ldots, m .
$$

Each map $y^{\alpha}\left(i+1^{\alpha} ; \cdot\right), i \in G_{k}(\alpha), \alpha=1, \ldots, m$, is $C^{1}$ on some neighborhood of 0 contained in $D_{1}$. On such a neighborhood,

$$
\left(\partial y^{\alpha} / \partial t_{\rho}\right)\left(i+1^{\alpha} ; 0\right)=A^{\alpha}(i) h^{\rho}(i)+F^{\alpha}\left(i, u^{\rho}(i)\right), \quad \rho=1, \ldots, \gamma+1 .
$$


Denote by $D$ a neighborhood of 0 in $E^{\gamma+1}$ on which each of the maps $y^{\alpha}\left(i+1^{\alpha} ; \cdot\right), i \in G_{k}(\alpha), \alpha=1, \ldots, m$, and $f_{0}(\hat{\sigma}(k ; \cdot))$ is $C^{1}$. Define the map

$$
\Theta \equiv\left(\Theta_{0},\left(\Theta_{i \alpha}\right)_{i \alpha}\right): D \times E^{\gamma+1} \rightarrow E^{n}
$$

by

$$
\begin{gathered}
\Theta_{0}\left(t_{0}, t\right) \stackrel{\Delta}{=} f_{0}(\hat{\sigma}(k ; t))+t_{0}-f_{0}\left(x^{*}(k)\right), \\
\Theta_{i \alpha}\left(t_{0}, t\right) \triangleq y^{\alpha}\left(i+1^{\alpha} ; t\right)-S^{\alpha}(i) \hat{\sigma}\left(i+1^{\alpha} ; t\right), \quad i \in G_{k}(\alpha), \alpha=1, \ldots, m .
\end{gathered}
$$

Then, $\Theta$ is $C^{1}$ and

$$
\Theta(0,0)=0
$$

Moreover, using Eqs. (11)-(12), it is easy to show that

$$
(\partial \Theta / \partial t)(0,0)=(-1)^{\gamma+2} \eta(\gamma+2) \delta^{\gamma+1} \neq 0 .
$$

By the classical implicit function theorem, there exist $\delta_{0}>0$ and a $C^{1}$-map $T \equiv\left(T_{1}, \ldots, T_{\gamma+1}\right): D \rightarrow E^{\gamma+1}$ satisfying

$$
T(0)=0 \quad \text { and } \Theta\left(t_{0}, T\left(t_{0}\right)\right)=0, \quad-\delta_{0}<t_{0}<\delta_{0} .
$$

Since $T$ is continuous, there exists $\delta_{0}^{1}>0, \delta_{0}^{1} \leq \delta_{0}$, such that, for each $\rho$ in $\{1, \ldots, \gamma+1\}$, if

$$
0<t_{0}<\delta_{0}^{1}
$$

then

$$
\left|T_{\rho}\left(t_{0}\right)\right|<(\gamma+1)^{-1} \text {. }
$$

Differentiating the second of Eqs. (14) and applying Cramer's rule yields expressions for the derivatives of the components of $T$ on $\left(-\delta_{0}, \delta_{0}\right)$. In particular,

$$
\left(d T_{\rho} / d t_{0}\right)(0)=\eta^{-1}(\gamma+2)^{-1}>0, \quad \rho=1, \ldots, \gamma+1 .
$$

It follows from the fundamental theorem of calculus that there exists $\delta_{0}^{2}>0$, $\delta_{0}^{2} \leq \delta_{0}^{1}$, such that, if

$$
0<t_{0}<\delta_{0}^{2}
$$

then

$$
T_{\rho}\left(t_{0}\right)>0, \quad \rho=1, \ldots, \gamma+1 .
$$

Choose $t_{0}$ satisfying

$$
0<t_{0}<\delta_{0}^{2}
$$


It is clear from the preceding that the components of $T\left(t_{0}\right)$ satisfy relations (13). Hence, there exists a map $u\left(\cdot ; T\left(t_{0}\right)\right): G \sim k \rightarrow U_{0}$, with $u\left(i ; T\left(t_{0}\right)\right) \in U(i), i \in G \sim k$, such that

$$
\begin{aligned}
& y^{\alpha}\left(i+1^{\alpha} ; T\left(t_{0}\right)\right)=f^{\alpha}\left(i, \sigma\left(i ; T\left(t_{0}\right)\right), u\left(i ; T\left(t_{0}\right)\right)\right), \\
& i \in G_{k}(\alpha), \quad \alpha=1, \ldots, m .
\end{aligned}
$$

Thus, for each pair $\alpha$ in $\{1, \ldots, \mathrm{m}\}, i$ in $G_{k}(\alpha)$, the condition

$$
\Theta_{i \alpha}\left(t_{0}, T\left(t_{0}\right)\right)=0
$$

implies that

$$
S^{\alpha}(i) \sigma\left(i+1^{\alpha} ; T\left(t_{0}\right)\right)=y^{\alpha}\left(i+1^{\alpha} ; T\left(t_{0}\right)\right)=f^{\alpha}\left(i, \sigma\left(i ; T\left(t_{0}\right)\right), u\left(i ; T\left(t_{0}\right)\right)\right) ;
$$

i.e., the pair $\left(\sigma\left(\cdot ; T\left(t_{0}\right)\right), u\left(\cdot ; T\left(t_{0}\right)\right)\right)$ is admissible. Moreover, since

$$
\Theta_{0}\left(t_{0}, T\left(t_{0}\right)\right)=0,
$$

it follows that

$I\left(\sigma\left(\cdot ; T\left(t_{0}\right)\right), u\left(\cdot ; T\left(t_{0}\right)\right)\right)-I\left(x^{*}, u^{*}\right) \equiv f_{0}\left(\sigma\left(k ; T\left(t_{0}\right)\right)\right)-f_{0}\left(x^{*}(k)\right)=-t_{0}<0$. In such a case, the pair $\left(x^{*}, u^{*}\right)$ cannot be optimal. The proof is complete.

Remarks. It is well known that some form of convexity hypothesis is needed to establish a global minimum principle for discrete-time systems (Ref. 20), except for a very restricted class of problems (Ref. 37). Simple counterexamples to Theorem 3.1 can be found if the convexity hypothesis (H3) is not satisfied (Ref. 15).

The notion of a derived cone has been used in the present paper as a conical approximation to the state constraint sets. This notion was discussed by Hestenes (Ref. 38) and was used by Hautus (Ref. 25) in developing a general discrete-time maximum principle. The definition used in the present paper differs slightly from those used by the above-named authors (which also differ slightly from each other); the minor differences are discussed in Ref. 15. In a recent notable paper (Ref. 39), Martin et al. give an exhaustive comparison of the many types of convex conical approximations that have appeared in the literature.

There is a natural correspondence between the discrete multiparameter minimum principle of the present paper and the minimum principle developed by Cesari in Ref. 12 for partial differential equations in Dieudonné-Rashevsky form. A detailed comparison is given in Ref. 15. 


\section{Applications}

Discrete multiparameter dynamic models, whether formulated ab initio as a discrete representation or obtained as a discretization of a continuum representation, frequently take the form of second-order or higher-order partial difference equations. These equations convey an insight into the underlying physical process being modeled, which may be obscured or destroyed by transforming the model into another representation purely for mathematical convenience. For example, one may produce some equivalent canonical set of first-order partial difference equations, or perhaps select an ordering of the independent discrete variables leading to an equivalent discrete-time system. The latter approach is often particularly undesirable; indeed, this fact has helped to motivate the development of discrete multiparameter linear system theory (Ref. 40).

As mentioned previously, most discrete multiparameter models encountered in applications can be expressed in the general form proposed in Section 2. The minimum principle of Section 3 can thus be interpreted in terms of a physically motivated higher-order discrete model. Quite general optimization problems using a discrete representation associated with hyperbolic (causal), parabolic (semicausal), and elliptic (noncausal) partial differential equation models have been treated in this manner (Ref. 15).

\section{References}

1. VAN DER VAART, H. R., A Comparative Investigation of Certain Difference Equations and Related Differential Equations: Implications for Model Building, Bulletin of Mathematical Biology, Vol. 35, pp. 195-211, 1973.

2. InNIs, G., Dynamic Analysis in "Soft Science" Studies: In Defense of Difference Equations, Mathematical Problems in Biology, Edited by P. Van den Driessche, Springer-Verlag, New York, New York, 1974.

3. GReENSPAN, D., Discrete Models, Addison-Wesley, Reading, Massachusetts, 1973.

4. GuCKenheimer, J., Oster, G., and IPAktchi, A., The Dynamics of Density Dependent Population Models, Journal of Mathematical Biology, Vol. 4, pp. 101-147, 1977.

5. Greenspan, D., Arithmetic Applied Mathematics, Pergamon, New York, New York, 1980.

6. Hsu, C. S., On Nonlinear Parametric Excitation Problems, Advances in Applied Mechanics, Edited by C. S. Yih, Academic Press, New York, New York, Vol. $17,1977$.

7. Gersho, A., and Gopinath, B., Charge-Routing Networks, IEEE Transactions on Circuits and Systems, Vol. CAS-26, pp. 81-92, 1979. 
8. JAIN, A. K., Partial Differential Equations and Finite Difference Methods in Image Processing, Part 1: Image Representation, Journal of Optimization Theory and Applications, Vol. 23, pp. 65-91, 1977.

9. JAIN, A. K., and JAIN, J. R., Partial Differential Equations and Finite Difference Methods in Image Processing, Part 2: Image Restoration, IEEE Transactions on Automatic Control, Vol. AC-23, pp. 817-834, 1978.

10. JAIN, A. K., Advances in Mathematical Models for Image Processing, Proceedings of the IEEE, Vol. 69, pp. 502-528, 1981.

11. BoSE, N. K., Editor, Special Issue on Multidimensional Systems, Proceedings of the IEEE, Vol. 65, No. 6, 1977.

12. Cesari, L., Optimization with Partial Differential Equations in DieudonnéRashevsky Form and Conjugate Problems, Archive for Rational Mechanics and Analysis, Vol. 33, pp. 339-357, 1969.

13. SuryanarayanA, M. B., Necessary Conditions for Optimization Problems with Hyperbolic Partial Differential Equations, SIAM Journal on Control, Vol. 11, pp. 130-147, 1973.

14. LUENBERGER, D. G., Dynamic Equations in Descriptor Form, IEEE Transactions on Automatic Control, Vol, AC-22, pp. 312-321, 1977.

15. HEGG, D. R., Necessary Conditions for Optimal Control of Systems Governed by Partial Difference Equations, University of Michigan, PhD Thesis, 1976.

16. ATTASI, S., Modelling and Recursive Estimation for Double Indexed Sequences, System Identification: Advances and Case Studies, Edited by R. K. Mehra and D. G. Lainiotis, Academic Press, New York, New York, 1976.

17. ROESSER, R. P., A Discrete State-Space Model for Linear Image Processing, IEEE Transactions on Automatic Control, Vol. AC-20, pp. 1-10, 1975.

18. FORNASINI, E., and MARCHESINI, G., State-Space Realization Theory of Two-Dimensional Filters, IEEE Transactions on Automatic Control, Vol. AC21, pp. 484-492, 1976.

19. BAUM, R. F., and CESARI, L., On a Recent Proof of Pontryagin's Necessary Conditions, SIAM Journal on Control, Vol. 10, pp. 56-75, 1972.

20. ROZONOÉR, L. I., The Maximum Principle of L.S. Pontryagin in Optimal System Theory, Part 3, Automation and Remote Control, Vol. 20, pp. 15171532, 1959.

21. Halkin, H., A Maximum Principle of the Pontryagin Type for Nonlinear Difference Equations, SIAM Journal on Control, Vol. 4, pp. 90-111, 1966.

22. Holtzman, J. M., Convexity and the Maximum Principle for Discrete Systems, IEEE Transactions on Automatic Control, Vol. AC-11, pp. 30-35, 1966.

23. Holtzman, J. M., and Halkin, H., Directional Convexity and the Maximum Principle for Discrete Systems, SIAM Journal on Control, Vol. 4, pp. 263-275, 1966.

24. Canon, M. D., Cullum, C. D., JR., and Polak, E., Theory of Optimal Control and Mathematical Programming, McGraw Hill, New York, New York, 1970.

25. Hautus, M. L. J., Necessary Conditions for Multiple Constraint Optimization Problems, SIAM Journal on Control, Vol. 11, pp. 653-669, 1973. 
26. LEE, K. Y., CHOW, S. N., and BARR, R. O., On the Control of Discrete-Time Distributed Parameter Systems, SIAM Journal on Control, Vol. 10, pp. 361-376, 1972.

27. Brockett, R. W., and Willems, J. L., Discretized Partial Differential Equations: Examples of Control Systems Defined on Modules, Automatica, Vol. 10, pp. 507-515, 1974.

28. ABDIKerIMOV, T., Optimal Processes in Discrete Systems with Distributed Parameters, Automation and Remote Control, Vol. 26, pp. 214-221, 1965.

29. Vostrova, Z. I., Optimal Processes in Sampled-Data Systems Containing Objects with Distributed Parameters, Automation and Remote Control, Vol. 27, pp. 767-779, 1966.

30. Vasil'ev, O. V., and Kirillova, F. M., Optimal Processes in Discrete Two-Parameter Systems, Soviet Mathematics Doklady, Vol. 8, pp. 790-793, 1967.

31. Fond, S., A Dynamic Programming Approach to the Maximum Principle of Distributed-Parameter Systems, Journal of Optimization Theory and Applications, Vol. 27, pp. 583-601, 1979.

32. Kung, S. Y., LÉvy, B. C., MORF, M., and KaIlath, T., New Results in 2-D Systems Theory, Part 2: 2-D State-Space Models-Realization and the Notions of Controllability, Observability, and Minimality, Proceedings of the IEEE, Vol. 65, pp. 945-961, 1977.

33. FornAsini, E., and MARCHEsInI, G., Doubly Indexed Dynamical Systems: State-Space Models and Structural Properties, Mathematical Systems Theory, Vol. 12, pp. 59-72, 1978.

34. CHAN, D. S. K., The Structure of Recursible Multidimensional Discrete Systems, IEEE Transactions on Automatic Control, Vol. AC-25, pp. 663-673, 1980.

35. WILLSKY, A., Relationships between Digital Signal Processing and Control and Estimation Theory, Proceedings of the IEEE, Vol. 66, pp. 996-1017, 1978.

36. EgGleston, H. G., Convexity, Cambridge University Press, Cambridge, England, 1969.

37. GABASOv, R., The Theory of Optimum Processes in Discrete Systems, USSR Computational Mathematics and Mathematical Physics, Vol. 8, pp. 99-123, 1968.

38. Hestenes, M. R., Calculus of Variations and Optimal Control Theory, Wiley, New York, New York, 1966.

39. MARTin, D. H., Gardner, R. J., and WAtkINS, G. G., Indicating Cones and the Intersection Principle for Tangential Approximants in Abstract Multiplier Rules, Journal of Optimization Theory and Applications, Vol. 33, pp. 515-537, 1981.

40. Woods, J. W. and Radewan, C. H., Kalman Filtering in Two Dimensions, IEEE Transactions on Information Theory, Vol. IT-23, pp. 473-482, 1977. 